\title{
ON THE RING OF LOCALLY BOUNDED NASH MEROMORPHIC FUNCTIONS
}

\author{
Wojciech Kucharz and Kamil RUSEK
}

We show that the ring of locally bounded Nash meromorphic functions on a connected $d$-dimensional Nash submanifold of $\mathbb{R}^{n}$ is a Prüfer domain and every finitely generated ideal in this ring can be generated by $d+1$ elements.

Moreover, every finitely generated ideal can be generated by $d$ elements if and only if the Nash manifold is noncompact.

\section{INTRODUCTION}

Let $M$ be a connected Nash submanifold of $\mathbb{R}^{n}$ of dimension $d$. The ring $\mathcal{N}(M)$ of all Nash functions on $M$ is an integral domain. (For background material on Nash manifolds and Nash functions the reader may refer to [1].) We call elements of the quotient field $\mathcal{L}(M)$ of $\mathcal{N}(M)$ Nash meromorphic functions on $M$.

A Nash meromorphic function $\varphi=f / g$ on $M$ is said to be locally bounded if for every point $x$ in $M$ there exist a neighbourhood $U_{x}$ of $x$ in $M$ and a positive real number $C_{\mathbf{x}}$ such that

$$
|f(y) / g(y)| \leqslant C_{x} \quad \text { for } \quad y \in U_{x} \backslash g^{-1}(0)
$$

(Of course, this definition does not depend on the choice of $f$ and $g$ in $\mathcal{N}(M)$ with $\varphi=f / g$.)

The set of all locally bounded Nash meromorphic functions on $M$ forms a subring of $\mathcal{L}(M)$, which we denote by $\mathcal{L}_{l b}(M)$. Clearly, one has

$$
\mathcal{N}(M) \subset \mathcal{L}_{l b}(M) \subset \mathcal{L}(M)
$$

It is well known that $\mathcal{N}(M)$ has many "good" algebraic properties. For example, $\mathcal{N}(M)$ is a Noetherian ring $[1$, Théorème 8.7.15].

\section{Received 4th January, 1996}

The first author was partially supported by NSF grant DMS-9503138. The second author was partially supported by KBN grant P03A06108. This note was prepared during the second author's nine-months stay at the Department of Mathematics and Statistics of the University of New Mexico in Albuquerque. He wishes to thank the Department of Mathematics and Statistics for the invitation and the agreeable hospitality.

Copyright Clearance Centre, Inc. Serial-fee code: 0004-9729/96 \$A2.00+0.00. 
If $d=1$, then one can easily verify that $\mathcal{N}(M)=\mathcal{L}_{\text {lb }}(M)$. For $d \geqslant 2$, the ring $\mathcal{L}_{l b}(M)$ is not Noetherian. Indeed, then there exist a sequence $\left\{a_{\nu}=\left(a_{\nu 1}, \ldots, a_{\nu n}\right)\right\}$ of points in $M$ and an index $k \in\{1, \ldots, n\}$ such that $a_{\nu k} \neq a_{\mu k}$ for $\nu \neq \mu$. Suppose, for simplicity, that $k=2$ and define

$f_{\nu}\left(x_{1}, \ldots, x_{n}\right)=\frac{\left(x_{1}-a_{\nu 1}\right)^{3}}{\left(x_{1}-a_{\nu 1}\right)^{2}+\left(x_{2}-a_{\nu 2}\right)^{2}}$ for $\left(x_{1}, \ldots, x_{n}\right) \in M$ and $\nu=1,2,3 \ldots$

Then each function $f_{\nu}$ is in $\mathcal{L}_{l b}(M)$ and it is easy to see that the sequence of ideals $\left(f_{1}\right) \subset\left(f_{1}, f_{2}\right) \subset\left(f_{1}, f_{2}, f_{3}\right) \subset \ldots$ in $\mathcal{L}_{l b}(M)$ is not stationary.

However, the ring $\mathcal{L}_{l b}(M)$ has some amusing algebraic properties.

Let us recall that a Prüfer domain is an integral domain whose every finitely generated fractional ideal is invertible. (See [3] for other characterisations of Prüfer domains.)

In this note we prove the following:

Theorem 1. $\mathcal{L}_{l b}(M)$ is a Prüfer domain.

THEOREM 2 .

(i) Every finitely generated ideal in $\mathcal{L}_{l b}(M)$ can be generated by $d+1$ elements.

(ii) Every finitely generated ideal in $\mathcal{L}_{l b}(M)$ can be generated by $d$ elements if and only if $M$ is noncompact.

\section{LOCALLY BOUNDED RATIONAL FUNCTIONS}

We need some preliminary results on locally bounded rational functions. Let $X$ be an affine irreducible nonsingular real algebraic variety of dimension $d$. Given a subset $A$ of $X$, we denote by $\mathcal{H}_{A}(X)$ the ring of rational functions on $X$ locally bounded on $A$ (that is, bounded in a neighbourhood of every point in $A$ ). If $U$ is a Zariski open subset of $X$, then $\mathcal{R}(U)$ will denote the ring of regular functions on $U$.

We shall identify in the sequel the rings $\mathcal{H}_{U}(X)$ and $\mathcal{H}_{U}(U)$ (clearly, the restriction homomorphism is an isomorphism of these rings).

Let $F$ be the field of rational functions on $U$ and let $\mathcal{A}$ be a finitely generated $\mathbb{R}$-subalgebra of $F$ whose quotient field is $F$. Then $\mathcal{H}_{U}(U)$ can be identified with the real holomorphy ring $H(F \mid \mathcal{A})$ of $F$ over $\mathcal{A}$. Hence the ring $\mathcal{H}_{U}(U)$ is a Prüfer domain and every finitely generated ideal of this ring can be generated by $d+1$ elements (see [2, Introduction and p.307] and the references given there; note that the results of [2] which are formulated in the language of schemes apply to our case of affine varieties).

LEMMA 2.1. Let $\varphi_{1}, \ldots, \varphi_{k}$ be in $\mathcal{H}_{A}(X)$. Then there exist an affine irreducible nonsingular real algebraic variety $Y$ of dimension $d$, a mapping $\pi: Y \rightarrow X$ which is 
a composition of finitely many algebraic blowing-ups with nonsingular centres, and a Zariski neighbourhood $V$ of $\pi^{-1}(A)$ in $Y$ such that $\varphi_{i} \circ \pi$ is in $\mathcal{R}(V)$ for $i=1, \ldots, k$.

ProOF: By Hironaka's resolution of singularities theorem [4], there exist a variety $Y$ with the above properties and a mapping $\pi: Y \longrightarrow X$, a composition of finitely many "good" blowing-ups, such that each function $\varphi_{j}$ has a normal form in a Zariski neighbourhood of every point of $Y$, that is, $\varphi_{j} \circ \pi=a_{j} z_{1}^{\alpha_{1}} \cdots z_{d}^{\alpha_{d}}$, where $z_{1}, \ldots, z_{d}$ are local parameters, $a_{j}$ is invertible and $\alpha_{1}, \ldots, \alpha_{d}$ are integers.

This implies that the set of points at which the functions $\varphi_{j} \circ \pi$ are not locally bounded is algebraic (because locally it is the union of sets of the form $\left\{z_{i_{1}}=\cdots=\right.$ $\left.z_{i_{p}}=0\right\}$ ). Therefore its complement $V$ is Zariski open and contains $\pi^{-1}(A)$. Of course, the function $\varphi_{i} \circ \pi$ is in $\mathcal{R}(V)$ for $i=1, \ldots, k$.

LemMa 2.2.

(i) $\mathcal{H}_{A}(X)$ is a Prüfer domain.

(ii) Every finitely generated ideal in $\mathcal{H}_{A}(X)$ can be generated by $d+1$ elements.

(iii) If $A$ contains no compact connected component of $X$, then every finitely generated ideal in $\mathcal{H}_{A}(X)$ can be generated by $d$ elements.

Proof: We know that $\mathcal{H}_{X}(X)$ is a Prüfer domain. Obviously, $\mathcal{H}_{A}(X)$ is an overring of $\mathcal{H}_{X}(X)$ and hence (i) follows by virtue of [3, Theorem 26.1].

Let $I$ be an ideal in $\mathcal{H}_{A}(X)$ generated by $\varphi_{1}, \ldots, \varphi_{k}$. Choose $\pi: Y \longrightarrow X$ and $V$ as in Lemma 2.1. Let $J$ be the ideal in $\mathcal{H}_{V}(Y)$ generated by $\varphi_{1} \circ \pi, \ldots, \varphi_{k} \circ \pi$. Then $J$ can be generated by $d+1$ elements. Note that

$$
\left(\pi^{-1}\right)^{*}: \mathcal{H}_{V}(Y) \ni \varphi \longrightarrow \varphi \circ \pi^{-1} \in \mathcal{H}_{A}(X)
$$

is a well-defined ring homomorphism and $\left(\pi^{-1}\right)^{*}(J) \mathcal{H}_{A}(X)=I$. Therefore $I$ can be generated by $d+1$ elements and (ii) holds.

Suppose now that $A$ contains no compact connected component of $X$. Then $\pi^{-1}(A)$ contains no compact connected component of $Y$ and we can assume that $V$ has the same property (we modify $V$ by removing a finite subset, if necessary). By [2, Theorem 1.1], every finitely generated ideal in $\mathcal{H}_{V}(Y)$ can be generated by $d$ elements, and (iii) follows.

Lemma 2.3. Assume that $A$ contains a compact connected component $C$ of $X$. Let $x_{0}$ be a point in $C$ and let

$$
I=\left\{f \in \mathcal{R}(X) \mid f\left(x_{0}\right)=0\right\}
$$

Then the ideal $I \mathcal{H}_{A}(X)$ cannot be generated by $d$ elements. 
ProOf: Let $\rho: X^{\prime} \longrightarrow X$ be the algebraic blowing-up of $X$ at. $x_{0}$ and let $J=$ $\rho^{*}(I) \mathcal{R}\left(X^{\prime}\right)$, where $\rho^{*}: \mathcal{R}(X) \longrightarrow \mathcal{R}\left(X^{\prime}\right)$ is the ring homomorphism induced by $\rho$.

First observe that

$$
J=\left\{g \in \mathcal{R}\left(X^{\prime}\right) \mid g\left(x^{\prime}\right)=0 \text { for } x^{\prime} \in \rho^{-1}\left(x_{0}\right)\right\} .
$$

This equality is a consequence of the definition of the blowing-up at $x_{0}$ and the localglobal principle.

Suppose now that $I \mathcal{H}_{A}(X)$ can be generated by $d$ elements. Then the ideal $J \mathcal{H}_{\rho^{-1}(A)}\left(X^{\prime}\right)$ can also be generated by $d$ elements, say $\varphi_{1}, \ldots, \varphi_{d}$. Let $f_{1}, \ldots, f_{k}$ be generators of $J$. We have

$$
f_{i}=\sum_{j=1}^{d} a_{i j} \varphi_{j}
$$

for some $a_{i j}$ in $\mathcal{H}_{p^{-1}(A)}\left(X^{\prime}\right)$. By Lemma 2.1, there exist $\pi: Y \longrightarrow X^{\prime}$, a composition of finitely many algebraic blowing-ups with nonsingular centres, and a Zariski neighbourhood $V$ of $\pi^{-1}\left(\rho^{-1}(A)\right)$ in $Y$ such that $a_{i j} \circ \pi$ and $\varphi_{j} \circ \pi$ belong to $\mathcal{R}(V)$ for $i=1, \ldots, k, j=1, \ldots, d$.

It follows that if $K=\pi^{*}(J) R(Y)$, where $\pi^{*}: \mathcal{R}\left(X^{\prime}\right) \longrightarrow \mathcal{R}(Y)$ is the ring homomorphism induced by $\pi$, then the ideal $K \mathcal{H}_{V}(Y)$ is generated by $d$ elements, namely $\varphi_{1} \circ \pi, \ldots, \varphi_{d} \circ \pi$. On the other hand, it follows from the proof of Lemma 3.5 in [2] and our remarks at the beginning of this section that the ideal $K \mathcal{H}_{V}(Y)$ cannot be generated by $d$ elements. This contradiction proves Lemma 2.3 .

\section{PROOFS OF THEOREMS 1 AND 2}

We can now return to the study of the ring $\mathcal{L}_{l b}(M)$ and prove our main results.

Proof of TheOREM 1: Let $I$ be a finitely generated fractional ideal of $\mathcal{L}_{l b}(M)$, say, $I=\left(f_{1} / g_{1}, \ldots, f_{k} / g_{k}\right) \mathcal{L}_{l b}(M)$, where $f_{i}, g_{i}$ are in $\mathcal{N}(M), i=1, \ldots, k$. By the theorem of Artin and Mazur [2, Théorème 8.4.4], there exist an irreducible nonsingular real algebraic set $X \subset \mathbb{R}^{m}$ of dimension $d$, an open semialgebraic subset $M^{\prime}$ of $X$, a Nash diffeomorphism $\sigma: M \rightarrow M^{\prime}$, and polynomial functions $p_{i}, q_{i}: X \rightarrow \mathbb{R}, i=$ $1, \ldots, k$, such that $p_{i} \circ \sigma=f_{i}, q_{i} \circ \sigma=g_{i}$ for $i=1, \ldots, k$. Let $I^{\prime}$ be the fractional ideal of $\mathcal{H}_{M^{\prime}}(X)$ generated by $p_{1} / q_{1}, \ldots, p_{k} / q_{k}$. Consider the ring homomorphism

$$
\sigma^{*}: \mathcal{H}_{M^{\prime}}(X) \ni p / q \longrightarrow p \circ \sigma / q \circ \sigma \in \mathcal{L}_{l b}(M)
$$

Note that $\sigma^{*}\left(I^{\prime}\right) \mathcal{L}_{l b}(M)=I$. Therefore the conclusion follows from Lemma 2.2(i) and the last equality. 
Proof of Theorem 2: (i) follows from Lemma 2.2(ii) and the proof of Theorem 1. Furthermore, the same argument shows that if $M$ is noncompact then every finitely generated ideal of $\mathcal{L}_{l b}(M)$ is generated by $d$ elements (use Lemma 2.2(iii)).

Assume now that $M$ is compact. Let $x_{0}$ be a point in $M$ and let

$$
I=\left\{f \in \mathcal{N}(M) \mid f\left(x_{0}\right)=0\right\}
$$

Suppose that $I \mathcal{L}_{l b}(M)$ can be generated by $d$ elements $\varphi_{1} / \psi_{1}, \ldots, \varphi_{d} / \psi_{d}$, where $\varphi_{j}, \psi_{j}$ are in $\mathcal{N}(M)$. By the Artin-Mazur theorem, there exist an irreducible nonsingular algebraic set $X \subset \mathbb{R}^{m}$, an open semialgebraic subset $M^{\prime}$ of $X$, a Nash diffeomorphism $\sigma: M \longrightarrow M^{\prime}$ and polynomial functions $p_{i}, q_{i}: X \longrightarrow \mathbb{R}$ such that $p_{i} \circ \sigma=\varphi_{i}$, $q_{i} \circ \sigma=\psi_{i}$. Obviously, $M^{\prime}$ is a compact connected component of $X$. Let $y_{0}=\sigma\left(x_{0}\right)$ and let

$$
I_{y_{0}}=\left\{f \in \mathcal{R}(X) \mid f\left(y_{0}\right)=0\right\} .
$$

By Lemma 2.3, the ideal $I_{y_{0}} \mathcal{H}_{M^{\prime}}(X)$ cannot be generated by $d$ elements. On the other hand, the ideal $I_{y_{0}} \mathcal{H}_{M^{\prime}}(X)$ is generated by $p_{1} / q_{1}, \ldots p_{d} / q_{d}$. Hence we obtain a contradiction, and Theorem 2 is proved.

Problem. Let $N$ be a connected real analytic manifold of dimension $d$ and let $\mathcal{M}_{\text {loc }}(N)$ be the ring of locally bounded meromorphic functions on $N$. Are the counterparts of Theorems 1 and 2 true for the ring $\mathcal{M}_{\text {loc }}(N)$ ?

\section{REFERENCES}

[1] J. Bochnak, M. Coste and M.-F. Roy, Géométrie Algébrique Réelle, Ergeb. Math. Grenzgeb. 12 (Springer-Verlag, Berlin, Heidelberg, New York, 1987).

[2] M.A. Buchner and W. Kucharz, 'On relative real holomorphy rings', Manuscripta Math. 63 (1989), 303-316.

[3] R. Gilmer, Multiplicative ideal theory, Pure and Applied Math. 12 (Marcel Dekker, New York, 1972).

[4] H. Hironaka, 'Resolution of singularities of an algebraic variety over a field of characteristic zero', Ann. of Math. 79 (1964), 109-326.

Department of Mathematics and Statistics University of New Mexico

Albuquerque NM 87131

United States of America

e-mail: kucharx@math.unm.edu
Institute of Mathematics

Jagiellonian University

Reymonta 4

30-059 Kraków

Poland

e-mail: rusek@im.uj.edu.pl 